\title{
Metastização Óssea da Calote Craniana: Uma Forma Rara de Apresentação de Cancro da Mama Metastizado

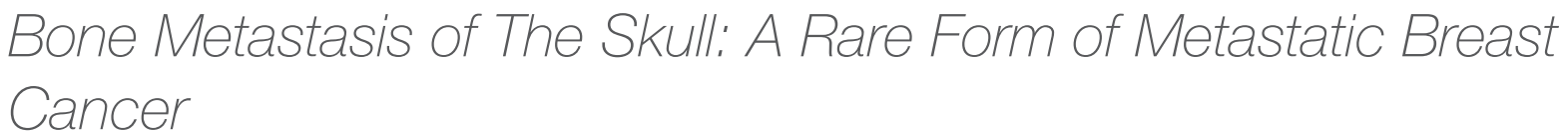

Catarina Duarte Santos (https://orcid.org/0000-0002-3545-2934), Tatiana Costa Pires (https://orcid.org/0000-00029048-2087), Rita Lizardo Grácio (htpps://orcid.org/0000-0001-9041-4028), Alcina Ponte (https://orcid.org/0000-00025468-9224)

Palavras-chave: Metástases Neoplásicas; Neoplasias da Mama; Neoplasias dos Ossos/secundárias.

Keywords: Bone Neoplasms/secondary; Breast Neoplasms; Neoplasm Metastasis.

O cancro da mama é o principal tipo de cancro mais frequentemente diagnosticado nas mulheres em Portugal. ${ }^{1}$ Apresenta uma incidência de aproximadamente 2 milhões de casos por ano em todo o mundo, e representa a principal causa de morte por neoplasia no sexo feminino. ${ }^{2} \mathrm{O}$ atingimento ósseo, hepático e pulmonar são as formas mais comuns de doença metastática. ${ }^{3}$ Quando ocorre metastização óssea, mais frequentemente ocorre metastização múltipla sendo os locais mais frequentes a coluna vertebral e os ossos longos. ${ }^{4}$

Apresenta-se o caso de uma doente de 76 anos de idade, previamente autónoma que foi referenciada ao Serviço de Urgência pelo Médico de Família por lesão intra-axial temporal direita, observada em tomografia computorizada crânio-encefálica, solicitada em contexto de desequilíbrio com mais de um ano de evolução. Ao exame objectivo destacava-se exame neurológico perfeitamente normal. Foi internada para estudo de lesão ocupante de espaço.

A ressonância magnética crânio-encefálica revelou lesões nodulares parenquimatosas rodeadas de edema vasogénico, em topografia periventricular lateral direita, parietal posterior esquerda, frontal direita e cerebelosa inferior esquerda; a nível da calote, na região parietal direita, uma outra lesão com erosão cortical óssea e associado componente tecidular tumoral epicraniano e intracraniano epidural (Fig.s 1- A, B e Fig.s 2 - C).

A cintigrafia óssea mostrou hipercaptação a nível da calote craniana, não se detetando alterações significativas em outras regiões do esqueleto.
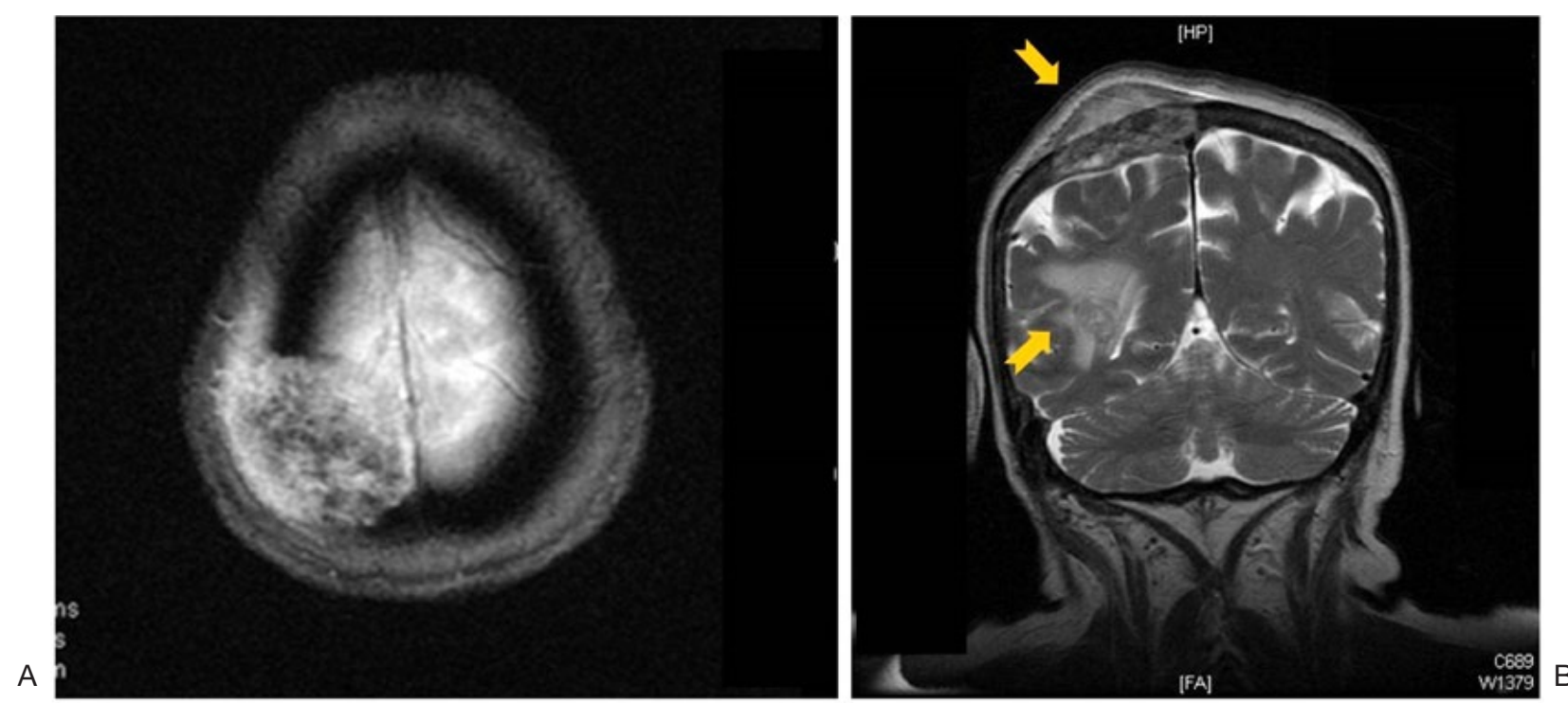

Figura 1: A - Lesão osteolítica da calote craniana; B - Lesão tumoral epicraniana e intracraniana epidural na região parietal direita e lesão parenquimatosa rodeada de edema vasogénico (plano coronal). 

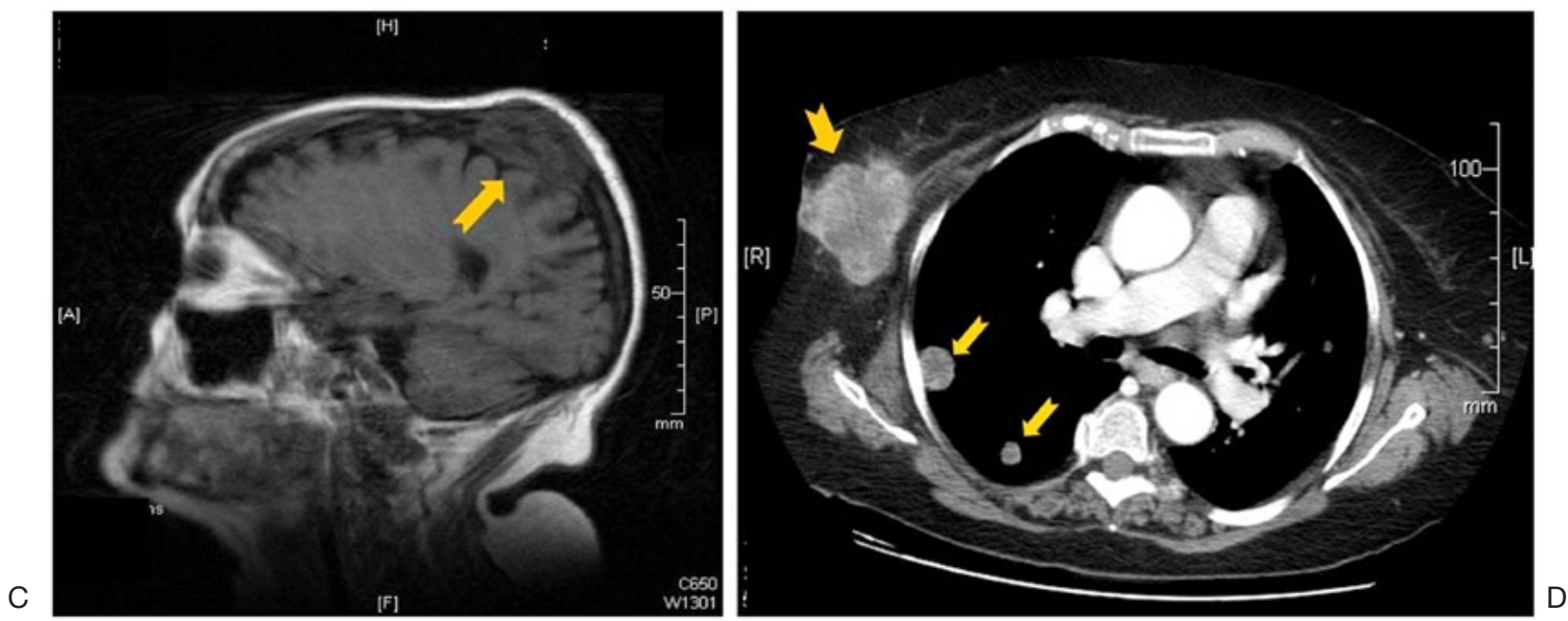

Figura 2: C - Lesão tumoral epicraniana e intracraniana na região parietal (plano sagital); D - Massa tumoral no quadrante superointerno da mama direita e metástases pulmonares.

A tomografia toraco-abdomino-pélvica revelou múltiplas lesões pulmonares nodulares dispersas em todos os segmentos, volumosas adenopatias axilares direitas e, no quadrante supero-interno da mama direita foi detectada volumosa massa de $50 \mathrm{~mm}$, de contornos bosselados, algo espiculados, compatível com provável neoplasia (Fig. 2 - D). Esta última lesão foi, então, submetida a biópsia que identificou carcinoma mamário invasivo.

Após o diagnóstico e estadiamento a doente foi orientada para Oncologia, tendo sido submetida a radioterapia holocraneana a título paliativo.

Apesar do quadro clínico tão fruste, tendo em conta o estadio avançado da doença, após cerca de 6 meses a doente faleceu.

\section{Responsabilidades Éticas}

Conflitos de Interesse: Os autores declaram a inexistência de conflitos de interesse na realização do presente trabalho.

Fontes de Financiamento: Não existiram fontes externas de financiamento para a realização deste artigo.

Confidencialidade dos Dados: Os autores declaram ter seguido os protocolos da sua instituição acerca da publicação dos dados de doentes.

Proteção de Pessoas e Animais: Os autores declaram que os procedimentos seguidos estavam de acordo com os regulamentos estabelecidos pelos responsáveis da Comissão de Investigação Clínica e Ética e de acordo com a Declaração de Helsínquia da Associação Médica Mundial. Proveniência e Revisão por Pares: Não comissionado; revisão externa por pares.

\section{Ethical Disclosures}

Conflicts of interest: The authors have no conflicts of interest to declare. Financing Support: This work has not received any contribution, grant or scholarship

Confidentiality of Data: The authors declare that they have followed the protocols of their work center on the publication of data from patients.
Protection of Human and Animal Subjects: The authors declare that the procedures followed were in accordance with the regulations of the relevant clinical research ethics committee and with those of the Code of Ethics of the World Medical Association (Declaration of Helsinki).

Provenance and Peer Review: Not commissioned; externally peer reviewed.

(c) Autor (es) (ou seu (s) empregador (es)) e Revista SPMI 2021. Reutilização permitida de acordo com CC BY-NC. Nenhuma reutilização comercial. (c) Author(s) (or their employer(s)) and SPMl Joumal 2021. Re-use permitted under CC BY-NC. No commercial re-use.

\section{Correspondence / Correspondência:}

Catarina Duarte Santos - catarinagdsantos@gmail.com

Serviço de Medicina, Centro Hospitalar de Leiria, Leiria, Portugal.

R. de Santo André, 2410-197 Leiria

Received / Recebido: 11/09/2020

Accepted / Aceite: 19/11/2020

Publicado / Published: 15 de março de 2021

\section{REFERÊNCIAS}

1. International Agency for Research on Cancer. Population Fact Sheets Portugal - WHO. [accessed June 2020] https://gco.iarc.fr/today/data/factsheets/ populations/620-portugal-fact-sheets.pdf.

2. Bray F, Ferlay J, Soerjomataram I, Siegel RL, Torre LA, Jemal A. Global cancer statistics 2018: GLOBOCAN estimates of incidence and mortality worldwide for 36 cancers in 185 countries. CA Cancer J Clin. 2018;68:394424. doi:10.3322/caac.21492

3. Wang R, Zhu Y, Liu X, Liao X, He J, Niu L. The Clinicopathological Features and Survival Outcomes of Patients with Different Metastatic Sites in Stage IV Breast Cancer. BMC Cancer. 2019:1-12. doi:10.21203/rs.2.11765/v1

4. Yang $Y, M a Y$, Sheng J, et al. A multicenter, retrospective epidemiologic survey of the clinical features and management of bone metastatic disease in China. Chin J Cancer. 2016;35(1):1-6. doi:10.1186/s40880-016-0102-6 